\title{
Face Recognition using Multi Region Prominent LBP Representation
}

\author{
Srinivasa Reddy $\mathbf{K}^{1}$, Vijaya Kumar $\mathbf{V}^{2}$, Venkata Krishna ${ }^{3}$ \\ ${ }^{1}$ Departement of Computer Science and Engineering, Institute of Aeronautical Engineering, Hyderabad, India \\ ${ }^{2}$ Departement of Computer Science and Engineering, Anurag Group of Institutions, Hyderabad, India \\ ${ }^{3}$ Departement of Computer Science and Engineering, Vidya Jyothi Institute of Technology, Hyderabad, India
}

\begin{tabular}{l} 
Article Info \\
\hline Article history: \\
Received Mar 29, 2016 \\
Revised Aug 25, 2016 \\
Accepted Sep 08, 2016 \\
\hline
\end{tabular}

\section{Keyword:}

Local and macro structures

Local binary pattern

Non uniform local binary

pattern

Recognition

Uniform local binary pattern

\begin{abstract}
Various face recognition methods are derived using local features among them the Local Binary Pattern (LBP) approach is very famous. The histogram techniques based on LBP is a complex task. Later Uniform Local Binary Pattern (ULBP) is derived on LBP, based on the bitwise transitions and ULBP's are treated as the fundamental property of texture. The ULBP approach treated all Non-Uniform Local Binary Patterns' (NULBP) into one miscellaneous label. Recently we have derived Prominent LBP (PLBP), Maximum PLBP (MPLBP) and Smallest PLBP (SPLBP). The PLBP consists of the majority of the ULBP's and some of the NULBP's. The basic disadvantage of these various variants of LBP's is they are basically local approaches and completely failed in representing features derived from large regions or macrostructures, which are very much essential for faces. This paper derives PLBP's on the large region. The rectangular region of this paper is assumed with a size of multiples of three and PLBPs are evaluated on dividing each region into multiple regions. The proposed Multi RegionPLBP (MR-PLBP) approach is tested on three facial databases namely Yale, Indian and AT\&T ORL. The experimental results show the proposed approach significantly outperforms the other LBP based face recognition methods.
\end{abstract}

Copyright $\odot 2016$ Institute of Advanced Engineering and Science. All rights reserved.

\section{Corresponding Author:}

Srinivasa Reddy,

Departement of Computer Science and Engineering,

Institute of Aeronautical Engineering,

Dundigal, Hyderabad-500043, TS, India.

Email: kondsreenu@gmail.com

\section{INTRODUCTION}

Face recognition is one of the significant and prominent research topics for the last two decades, due to its potential applications and challenges. Face recognition deals with recognition of a test facial image from a feature library or data set of facial images. The researchers in image processing and facial image analytics of big data are showing tremendous interest because of its wide range of applications like human face interaction, surveillance in public and private military and other areas in various legal and property issues to identify the proper person. The face identification accuracy significantly decreases when obtained images do not have adequate quality either due to a variety of facial expressions, subject's alignment problem to the camera, gaze deviations or facial hair [1-5].

The human face can be identified based on the local and regional attributes. Today, the challenge in face recognition is, how to identify and capture this information. The Local Binary Pattern (LBP) is a powerful local descriptor for texture analysis and also extensively used in various applications [6]. The local information derived from LBP is used widely in facial analysis especially for the purpose of face recognition [7-13] age classification [14], [15-17] and facial expression recognition [18]. Few researchers 
used pre-processing methods to improve classification performance [12], [19] on LBP because it is more prone to noise.

The fundamental LBP operator has small spatial support area and they completely fail in capturing large scale structure (macrostructure) that may be the dominant feature of faces. In the literature, the Uniform Local Binary Pattern (ULBP) are used for classification and recognition purpose because they contain fundamental properties of the texture and ignored the majority of Non Uniform Local Binary Pattern (NULBP) as miscellaneous. The ULBPs alone may not describe stochastic attributes and characteristics of texture efficiently. Recently we have proposed a new variant of LBP called Prominent LBP (PLBP) that captures a set of ULBPs and a set of NULBPs. To capture local and regional information of faces with a uniformity of region and sub-region size the present paper proposes MR-PLBP. In the proposed method, the average value of each sub-region is converted into the grey level value of neighboring pixels. That's why the proposed method is more robust. The proposed MR-PLBP with various variants of PLBP is different from the other multi-block approaches because they have used uniform and other variants of LBP features [20].

The present paper is organized as follows. Section 2 describes the related work. The section 3 and 4 presents the methodology and results and discussion. Section 5 presents the conclusions.

\section{RELATED WORK}

\subsection{Local Binary Pattern (LBP)}

In the original LBP, introduced by the Ojala [6] a thresholding process between the grey level values of the central pixel and each of the neighborhood pixels on a $3 * 3$ window converts the neighboring pixel values into a binary value. The binary weights are multiplied with the binary value and sum of these values results as LBP code or weight as shown in Figure 1.

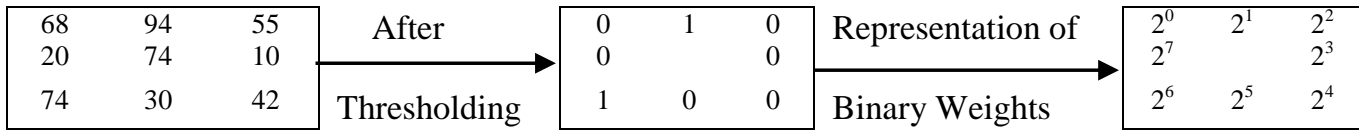

(c)

Figure 1. Representation of Basic LBP code (a) $3 * 3$ Neighborhood, (b) LBP Values after Thresholding, (c) Representation of LBP Weights, (d) LBP Code

The LBP Code can also be derived from the equation 1 [21]

$$
L B P-C o d e=\sum_{i=0}^{7} S\left(g_{i}-g_{c}\right) * 2^{i}
$$

where $g_{i}$ and $g_{c}$ represent the grey level values of the neighboring and central pixel on a $3 * 3$ neighborhood, $S$ represents the sign function, where

$$
S(x)=\left\{\begin{array}{l}
1 \text { if } x \geq 0 \\
0 \text { if } x<0
\end{array}\right.
$$

The Uniform Local Binary Pattern (ULBP) is derived on the LBP to improve the performance and to reduce overall features. The ULBP provides a majority of patterns: $90 \%$ for $(8,2)$ type LBP; $70 \%$ for $(16,2)$ type LBP [21], that's why they are treated as fundamental local texture properties of the image and researchers considered the NULBPs as miscellaneous. There will be 58 ULBPs and 198 NULBPs on a basic LBP i.e. $(8,1)$. This number of NULBPs increases drastically as we increase the size of the neighborhood or the number neighboring pixels.

\subsection{Prominent Local Binary Patten (PLBP)}

Many researchers expressed their views on the capability of ULBP and NULBP in terms of texture image analysis, recognition etc., some researchers [21-25] explored extensively NULBP's and derived a few NULBP and ULBP in a feature vector and conducted experiments. H. Zhou et al. [23] suggested that ULBP alone do not describe the stochastic attributes of texture efficiently. As a result, texture primitive information 
represented by these patterns is lost, especially when large neighborhoods are considered. This single pattern makes the uniform patterns sensitive to noise. An extended version of LBP operator (LBP extend) is also proposed in the literature [23], which tried to use more than one bin for describing non-uniform patterns and to reduce the effect of noise. Several other attempts were also made in the literature to use non-uniform patterns to overcome the limitation of the standard LBP [26-27], [9], [22], [13]. Some of the methods extracted rotation invariant non-uniform patterns [27], [9], [22].

The present research argues that some useful information can be obtained by using NULBPs. The major problem is what kind or type of NULBPs to be selected from the large set of NULBPs. So far there is no mechanism that derives the majority of the ULBPs and a few of NULBPs as one set. All the above researchers considered some NULBP in their own way. This has lead lot ambiguity. To overcome this ambiguity and to give a systematic way of selecting NULBPs the present paper utilized our previous derivation called Prominent Local Binary Pattern (PLBP) [8]. The interesting feature of PLBP is that it contains a set of ULBPs and NULBPs. The Prominent Local Binary Pattern (PLBP) considers the transition that occurs after two or more consecutive zeros immediately followed by two or more consecutive ones and vice versa, in a circular manner. For example, the LBP code 35 constitutes the PLBP and the LBP code 96 forms a Non Prominent Local Binary Pattern (NPLBP).

The PLBP contains a total of 92 patterns out of 256 of $L B P$ on a $(P, R)=(8,1)$. The PLBP contains 40 ULBPs out of 58 and 52 NULBPs out of 198. This means the PLBP discards 18 ULBPs and 146 NULBPs into one label called "miscellaneous". For the efficient face recognition system, the present paper derived two variants of PLBP, namely Maximum Prominent Local Binary Pattern (MPLBP) formed from PLBP U ULBP and Small Prominent Local Binary Pattern (SPLBP) formed from PLBP $\cap U L B P$ on facial images. The SPLBP and MPLBP contain a total of 40 and 110 patterns respectively on a $3 \times 3$ neighborhood. The following Table 1 shows the relationship between various variants of LBP i.e. ULBP, NULBP, PLBP, NPLBP, MPLBP and SPLBP.

Table 1. Relationship between Various Variants of LBP

\begin{tabular}{ccccccccc}
\hline S. No. & BINARY & $\begin{array}{c}\text { DECIMAL } \\
\text { PALUE }\end{array}$ & ULBP & NULBP & PLBP & NPLBP & MPLBP & SPLBP \\
\hline 1 & 00000000 & 0 & $\sqrt{ }$ & $\mathrm{X}$ & $\mathrm{X}$ & $\sqrt{ }$ & $\sqrt{ }$ & $\mathrm{X}$ \\
2 & 00000110 & 6 & $\sqrt{ }$ & $\mathrm{X}$ & $\sqrt{ }$ & $\mathrm{X}$ & $\sqrt{ }$ & $\sqrt{ }$ \\
3 & 00100011 & 35 & $\mathrm{X}$ & $\sqrt{ }$ & $\sqrt{ }$ & $\mathrm{X}$ & $\sqrt{ }$ & $\mathrm{X}$ \\
4 & 00111110 & 60 & $\sqrt{ }$ & $\mathrm{X}$ & $\sqrt{ }$ & $\mathrm{X}$ & $\sqrt{ }$ & $\sqrt{ }$ \\
5 & 01101111 & 111 & $\mathrm{X}$ & $\sqrt{ }$ & $\mathrm{X}$ & $\sqrt{ }$ & $\mathrm{X}$ & $\mathrm{X}$ \\
6 & 10110001 & 177 & $\mathrm{X}$ & $\sqrt{ }$ & $\sqrt{ }$ & $\mathrm{X}$ & $\sqrt{ }$ & $\mathrm{X}$ \\
7 & 11111011 & 251 & $\sqrt{ }$ & $\mathrm{X}$ & $\mathrm{X}$ & $\sqrt{ }$ & $\sqrt{ }$ & $\mathrm{X}$ \\
8 & 11111111 & 255 & $\sqrt{ }$ & $\mathrm{X}$ & $\mathrm{X}$ & $\sqrt{ }$ & $\sqrt{ }$ & $\mathrm{X}$ \\
\hline
\end{tabular}

The union of PLBP and ULBP (PLBP U ULBP) contains a total of 110 patterns out of which 58 are ULBPs and 52 are NULBPs. This set is named as Maximum Prominent Local Binary Pattern (MPLBP) [8]. The MPLBP treats the remaining 146 NULBPs as a miscellaneous set. The intersection of PLBP and ULBP (PLBP $\cap U L B P)$ is named as the Smallest Prominent Local Binary Pattern (SPLBP) [8]. The SPLBP contains a total of 40 patterns out of which all 40 are ULBPs and it contains zero NULBPs. The SPLBP treats the remaining 216 LBPs (which contain 18 ULBPs and 198 NULBPs) as a miscellaneous set.

\section{MULTI REGION PROMINENT LOCAL BINARY PATTERN (MR-PLBP)}

The basic LBP operators with any $(\mathrm{P}, \mathrm{R})$ (where $\mathrm{P}$ corresponds to the number of neighboring pixels on a circle of radius of $\mathrm{R}$ ) only capable of extracting features on small spatial neighborhood i.e. micro level features and thus they fail in capturing larger scale structures or macrostructures which are also dominant on faces. Further, the grey level comparison may prone to noise when differences of grey values of pixels are very small or equal [28-29]. To overcome this present paper proposes Multi Region- Prominent Local Binary Pattern (MR-PLBP). The main advantage of the MR-PLBP is the size of the block and sub-blocks are fixed (multiples of 3). The previous researchers derived only LBP or ULBP features are derived from multi blocks by treating all NULBPS of the region as miscellaneous. In the proposed MR-PLBP the size of the region is fixed as $R^{*} \mathrm{~S}$ where $\mathrm{R}$ and $\mathrm{S}$ should be multiples of three. The region of size $\mathrm{R} * \mathrm{~S}$ is subdivided into nine subregions of size $N^{*} M$ where $N=R / 3$ and $M=S / 3$. This gives the uniformity in the formation of MR-PLBP. The other advantage of the present method is it estimates the various variants of PLBP on multi regions.

The each multi region is represented by a single value and the value of each multi region is the average grey level value of the pixels of that sub-region. A binary code is derived from each sub-region by 
comparing its value with the value of the middle sub- region. The circular bitwise transition of this sub- region neighborhood derives PLBPs on the multi region. The scalar values i.e. average pixel grey level values of each sub-region of size $\mathrm{N}^{*} \mathrm{M}$ can be computed very efficiently from the integral image [30]. Therefore MR-PLBP features extraction process is very fast. However, it only incurs a little more cost when compared to basic LBP operator $(8,1)$. Even as ' $\mathrm{P}$ ' increases the basic LBP feature extraction becomes costlier. The basic parameters $\mathrm{R}$ and $\mathrm{S}$ of MR-PLBP influence the overall structure of the features. If $\mathrm{R}$ and $\mathrm{S}$ are small then MR-PLBP captures only the local features and when $\mathrm{R}$ and $\mathrm{S}$ are large (especially $\mathrm{R}$ and $S>=9$ ) the MR-PLBP captures both micro and macro structure features and especially the average grey level values of sub-regions $\mathrm{N}^{*} \mathrm{M}$ overcomes the noise effect, makes MR-PLBP as robust, and provides large scale information in addition to micro level information. The MR-PLBP mechanism on a region size ' $S$ ' of $9 * 9$ is shown in Figure 2, the block sizes are $3 * 3$.

\begin{tabular}{|lllllllll|}
\hline 25 & 24 & 50 & 45 & 22 & 33 & 18 & 19 & 24 \\
55 & 40 & 65 & 73 & 17 & 56 & 31 & 32 & 12 \\
80 & 75 & 45 & 55 & 45 & 68 & 28 & 27 & 34 \\
14 & 21 & 22 & 24 & 50 & 40 & 29 & 24 & 13 \\
19 & 23 & 24 & 40 & 35 & 23 & 17 & 28 & 11 \\
18 & 28 & 29 & 43 & 25 & 26 & 45 & 19 & 39 \\
31 & 32 & 12 & 24 & 49 & 26 & 22 & 25 & 45 \\
28 & 27 & 34 & 73 & 87 & 56 & 37 & 55 & 53 \\
22 & 30 & 45 & 20 & 12 & 13 & 45 & 80 & 34 \\
\hline
\end{tabular}

\begin{tabular}{|lll|}
\hline 51 & 46 & 25 \\
22 & 34 & 25 \\
29 & 40 & 44 \\
\hline
\end{tabular}

(b)

\begin{tabular}{|lll|}
\hline 1 & 1 & 0 \\
0 & & 0 \\
0 & 1 & 1 \\
\hline
\end{tabular}

$(51)_{10}$

(a)

(c)

(d)

Figure 2. Multi Region Prominent Local Binary Pattern (MR-PLBP) Code Generation, (a) Division of Region of Size 9*9 into '9' Sub Regions of 3*3 (b) Representation of Average Values of '9' Sub-Region of 3*3 (c) Representation r-Sub-Regions with Binary Values (d) MR-PLBP Code

The MR-PLBP code is evaluated in the same way as represented in Equation 1. The Figure 2 clearly shows the representation of large structures or macrostructures by MR-PLBP. The resulting binary patterns as features of MR-PLBP can detect diverse image structures such as lines, edges, spots, corners at different scale and location. There will be fewer numbers of MR-PLBP code features when compared to basic LBP. A basic LBP will generate $(\mathrm{N}-1) *(\mathrm{M}-1)$ LBP codes, whereas an MR-PLBP with a region size of $\mathrm{R} * \mathrm{~S}$ generates a total number of $(\mathrm{N} * \mathrm{M}) /(\mathrm{R} * \mathrm{~S}) \mathrm{LBP}$ codes in a non-overlapped manner.

\section{RESULTS AND DISCUSSION}

The proposed MR-PLBP used three different databases i.e. Yale, Indian and AT\&T ORL. The present paper considered 120 facial images out of 15 persons with 11 different facial expressions per person as training set from Yale database [31]. The present paper also considered 472 facial images as a training set from Indian database [32]. These 472 facial images correspond to 59 different individuals of both male and female, and on each individual 11 different expressions of Indian database. The present paper also considered 320 facial images as a training set from AT\&T ORL database [33] for face recognition. The present paper performed experiments by considering two cases for test database.

Test Case 1: In case1 the remaining leftover facial images of the above three databases (which are not considered for the training set) are considered as test images. Test Case 2: In the second case the present paper considered the test images as a combination of leftover and training database images.

For efficient face recognition, the present paper evaluated histograms of LBP, ULBP, PLBP, MPLBP and SPLBP with different region sizes on each individual facial image and placed in the training database. In a similar way the above histograms are evaluated for test facial image and the face recognition is evaluated based on Chi-Square distance method as given in Equation 2.

$$
R(d, t)=\min \left(\sum_{i=1}^{n}\left(\left(d_{i}-t_{i}\right)^{2} /\left(d_{i}+t_{i}\right)\right) / 2\right)
$$


where $\mathrm{d}$, $\mathrm{t}$ are two image features (histogram vectors) and $\mathrm{R}(\mathrm{d}, \mathrm{t})$ is the histogram distance for recognition.

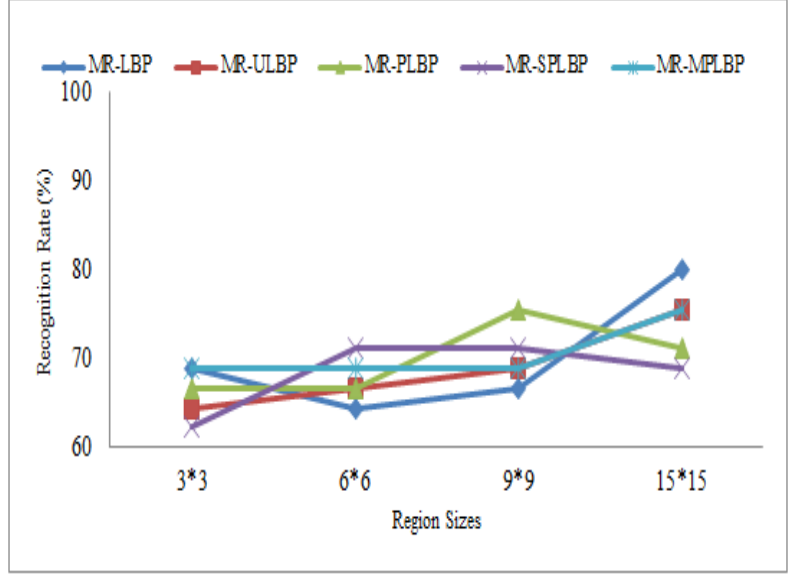

Figure 3. Face Recognition Rate for Yale Database for Test Case 1

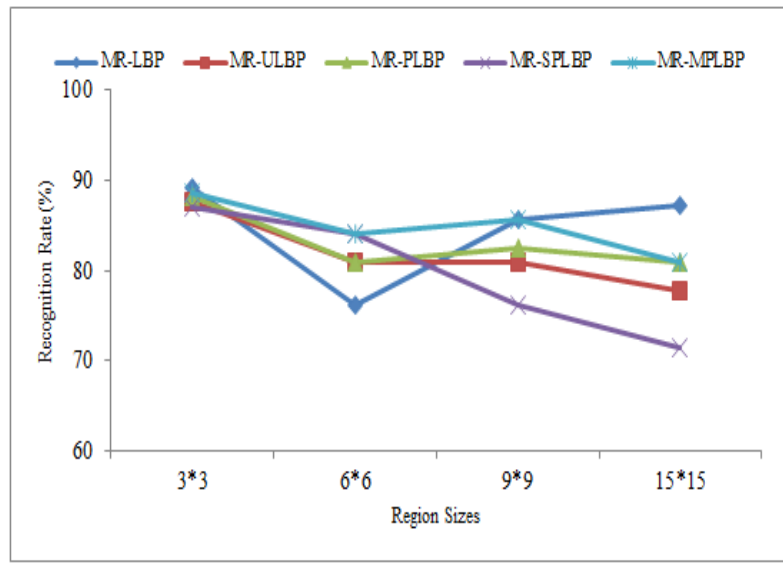

Figure 5. Face Recognition Rate for Indian Database for Test Case 1

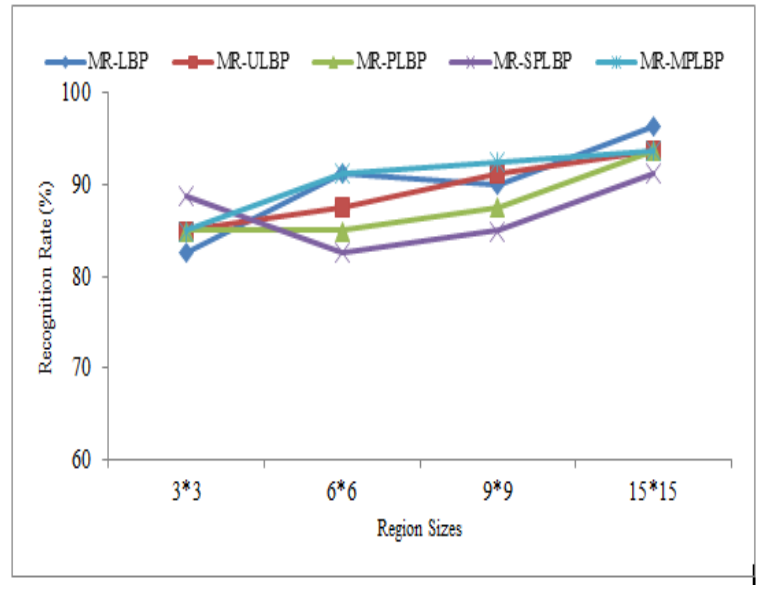

Figure 4. Face Recognition Rate for AT\&T ORL Database for Test Case 1

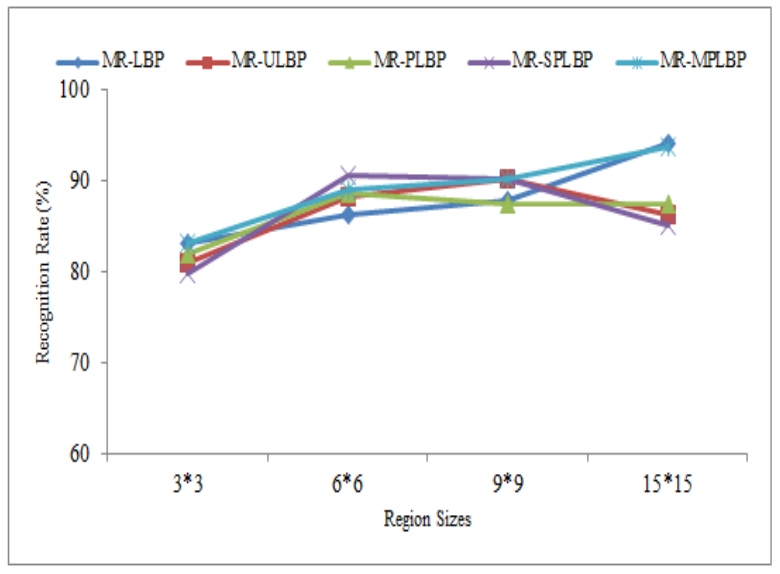

Figure 6. Face Recognition Rate for Yale Database for Test Case 2

The graphs of Figure 3, Figure 4, and Figure 5 shows the face recognition rate for Yale, AT\&T ORL and Indian databases for Test Case 1 with different region sizes. The same is also represented in graphs of Figure 6, Figure 7 and Figure 8 for Test Case 2.

The following factors are noted down from the graphs of figures from Figure 3 to Figure 8 . In the above graphs, the region size of $3 * 3$ represents the basic representation of LBP with $(8,1)$.

1. As the macro region increases the facial recognition rate increases slightly by the proposed MR PLBP and its variants i.e. MR-MPLBP and MR-SPLBP. This clearly reflects the fact that macro structure features are dominant in facial images and they are well captured by the proposed MR-PLBP, MR-SPLBP, and MR-MPLBP.

2. The face recognition rate for AT\&T ORL and Indian databases are high when compared to Yale database for case 1 and case 2. This is because the Yale database is prone to noise and illumination effects. 


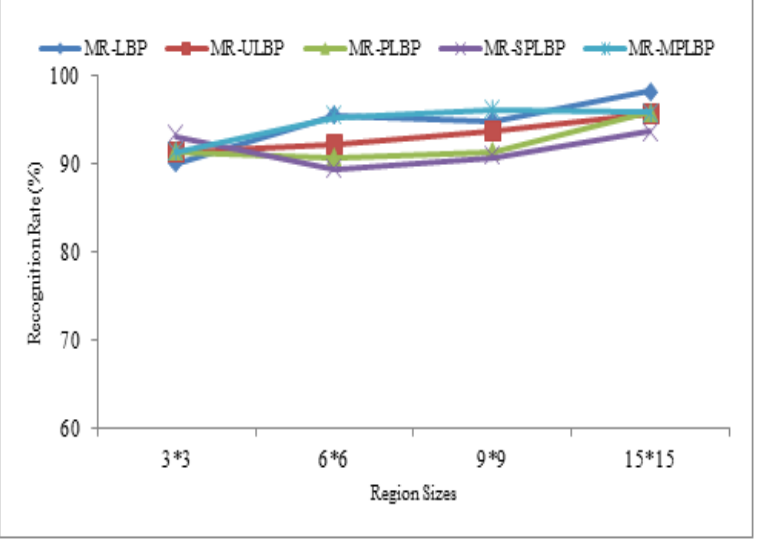

Figure 7. Face Recognition Rate for AT\&T ORL Database for Test Case 2

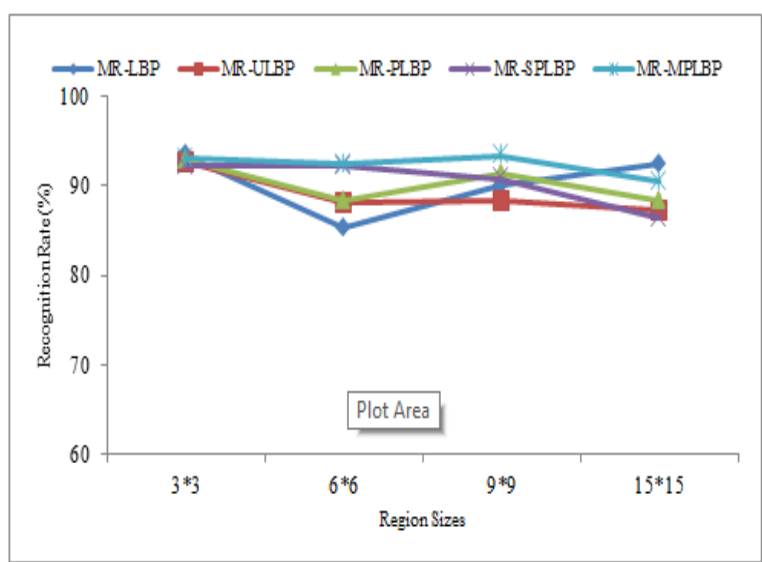

Figure 8. Face Recognition Rate for Indian Database for Test Case 2

\section{CONCLUSIONS}

In this paper, we proposed Multi Region Prominent Local Binary Pattern (MR-PLBP), MR-MPLBP and MR-SPLBP as a descriptor for face recognition to reflect the uniform appearance of the facial images. The Local Binary Pattern (LBP) is too local to be robust. Uniform patterns may not remain the same as those defined by Ojala et al [6] due to noise and they may not represent properly the stochastic information of textures. To describe fundamental and stochastic attributes efficiently, the present paper derived PLBP, MPLBP and SPLBP on the macro structures. Feature extraction for MR-PLBP is very fast using integral images. As the macro region increases the facial recognition rate increases slightly by the proposed MR-PLBP and its variants. This clearly reflects the fact macro structure features are dominant in facial images and they play a crucial role in face recognition than microstructure features. Moreover, our face recognition approach MR-SPLBP has shown very good performance on all databases with fewer uniform patterns and therefore it is more suitable for real time applications.

\section{REFERENCES}

[1] P. Dreuw, P. Steingrube, H. Hanselmann, H. Ney, and G. Aachen, "SURF-Face: Face Recognition under Viewpoint Consistency Constraints", In BMVC, pp. 1-11, 2009.

[2] X. Lu, and A.K. Jain, "Integrating range and texture information for 3D face recognition", Application of Computer Vision, Seventh IEEE Workshop, Vol. 1, pp. 156-163, Jan. 2005.

[3] J. Ruiz-del-Solar, R. Verschae, and M. Correa, "Recognition of faces in unconstrained environments: a comparative study", EURASIP Journal on Advances in Signal Processing, Vol. 2009, No. 1, pp. 1-20, Mar. 2009.

[4] J. Wright, and G. Hua, "Implicit elastic matching with random projections for pose-variant face recognition", Computer Vision and Pattern Recognition-IEEE Conference, pp. 1502-1509, Jun.2009.

[5] G. Zhao, and M. Pietikainen, "Dynamic texture recognition using local binary patterns with an application to facial expressions", Pattern Analysis and Machine Intelligence, IEEE Transactions, vol. 29, no. 6, pp. 915-928, Jun. 2007.

[6] T. Ojala, M. Pietikainen, and D. Harwood, "A comparative study of texture measures with classification based on featured distributions", Pattern recognition, Vol. 29, No. 1, pp. 51-59, Jan. 1996.

[7] T. Ahonen, A. Hadid, and M. Pietikainen, "Face description with local binary patterns: Application to face recognition", Pattern Analysis and Machine Intelligence, IEEE Transactions, vol. 28 no. 12, pp. 2037-2041, Dec. 2006.

[8] V. Vijaya Kumar, K. Srinivasa Reddy, and V. Venkata Krishna. "Face Recognition using Prominent LBP Model", International Journal of Applied Engineering Research, Vol. 10, No. 2, pp. 4373-4384, Mar. 2015.

[9] S. Liao, and A.C. Chung, "Face recognition by using elongated local binary patterns with average maximum distance gradient magnitude", Computer Vision-Springer, pp. 672-679, Nov. 2007.

[10] L. Nanni, and A. Lumini, "Region Boost learning for 2D+3D based face recognition", Pattern Recognition Letters, Vol. 28, No. 15, pp. 2063-2070, Nov. 2007.

[11] L. Nanni, S. Brahnam, and A. Lumini, "A local approach based on a Local Binary Patterns variant texture descriptor for classifying pain states", Expert Systems with Applications, Vol. 37, No. 12, pp. 7888-7894, Dec. 2010.

IJECE Vol. 6, No. 6, December $2016: 2781-2788$ 
[12] W. Zhang, S. Shan, W. Gao, X. Chen, and H. Zhang, "Local gabor binary pattern histogram sequence (lgbphs): A novel non-statistical model for face representation and recognition", Computer Vision, Tenth IEEE International Conference, Vol. 1, pp. 786-791, Oct. 2005.

[13] Sabina Yasmin, Md. Masud Rana, "Performance Study of Soft Local Binary Pattern over Local Binary Pattern under Noisy Images", International Journal of Electrical and Computer Engineering (IJECE), Vol. 6, No. 3, pp. 1161-1167, June 2016.

[14] M. Chandra Mohan, V. Vijaya Kumar, and B. Sujatha, "Classification of child and adult based on geometric features of face using linear wavelets", International Journal of Signal Processing, Image Processing and Pattern Recognition (IJSIP), vol. 1, no. 3, pp. 211-220, May 2010,

[15] A. Gunay, and V.V. Nabiyev, "Automatic age classification with LBP", Computer and Information Sciences, 23rd International Symposium, pp. 1-4, Oct. 2008.

[16] V.V. Kumar, J.S. Kiran, and V.H. Chandana, "An Effective Age Classification Using Topological Features Based on Compressed and Reduced Grey Level Model of the Facial Skin", International Journal of Image, Graphics and Signal Processing (IJIGSP), vol. 6, No. 1, pp. 9-17, Nov. 2013.

[17] B.E. Reddy, P.C.S. Reddy, and V.V. Kumar, "Texton Based Shape Features on Local Binary Pattern for Age Classification", International Journal of Image, Graphics and Signal Processing, Vol. 4, No. 7, pp. 54-60, Jul. 2012.

[18] D.C. He, and L. Wang, "Texture unit, texture spectrum, and texture analysis", Geoscience and Remote Sensing, IEEE Transactions, Vol. 28, no. 4, pp. 509-512, 1990.

[19] [19] L. Nanni, and A. Lumini, "Local binary patterns for a hybrid fingerprint matcher", Pattern recognition, vol. 41, no.11, pp. 3461-3466, Nov. 2008.

[20] T. Ahonen, A. Hadid, and M. Pietikainen, "Face recognition with local binary patterns", In Computer vision-eccv 2004, pp. 469-481, 2004.

[21] Z. Guo, L. Zhang, D.Zhang, and X. Mou, "Hierarchical multiscale LBP for face and palmprint recognition", Image Processing, 17th IEEE International Conference, pp. 4521-4524, Sept. 2010,

[22] T. Ojala, M. Pietikainen, and T. Maenpaa, "Multiresolution gray-scale and rotation invariant texture classification with local binary patterns", Pattern Analysis and Machine Intelligence, IEEE Transactions, Vol. 24, No. 7, pp. 971987, Jul. 2002.

[23] S. Liao, M. W. Law, and A. Chung, "Dominant local binary patterns for texture classification", Image Processing, IEEE Transactions, Vol. 18, No. 5, pp. 1107-1118, May, 2009.

[24] H. Zhou, R. Wang, and C. Wang, "A novel extended local-binary-pattern operator for texture analysis", Information Sciences, Vol. 178, No. 22, pp. 4314-4325, Nov. 2008.

[25] K. Srinivasa Reddy, V. Venkata Krishna, and B. Eswara Reddy, "Facial Recognition using the combination of significant non uniform local binary patterns", Graphics Vision and Image Processing, Vol. 15, No. 1, pp 1-8, Jun. 2015 ,

[26] K.S. Reddy, V.V. Krishna, and V.V. Kumar, "A Method for Facial Recognition Based On Local Features", International Journal of Mathematics \& Computation, Vol. 27, Iss. 3, pp.98-109, 2015.

[27] A. Fathi, and A.R. Naghsh-Nilchi, "Noise tolerant local binary pattern operator for efficient texture analysis", Pattern Recognition Letters, vol. 33, no. 9, pp. 1093-1100, Jul. 2012.

[28] M. Heikkila, M. Pietikainen, and C. Schmid, "Description of interest regions with local binary patterns", Pattern recognition, vol. 42, no. 3, pp. 425-436, Mar. 2009

[29] K. Srinivasa Reddy, V. Vijaya Kumar, B. Eswara Reddy, "Face recognition based on texture features using local ternary patterns", International Journal of Image, Graphics and Signal Processing, Vol. 7, No. 10, pp. 37-46, 2015.

[30] X. Tan, and B. Triggs, "Enhanced local texture feature sets for face recognition under difficult lighting conditions", Image Processing, IEEE Transactions, Vol. 19, No. 6, pp. 1635-1650, Jun. 2010

[31] P. Viola, and M. Jones, "Rapid object detection using a boosted cascade of simple features", Computer Vision and Pattern Recognition, IEEE Computer Society Conference, Vol. 1, pp. I-511- I-518, 2001.

[32] P.N. Belhumeur, and D.J. Kriegman, "The Yale face database. URL: http://cvc. yale. edu/projects/yalefaces/yalefaces.html", vol. 1, no. 2, pp. 4, 1997.

[33] V. Jain, and A. Mukherjee, "The Indian face database", 2002.

\section{BIOGRAPHIES OF AUTHORS}

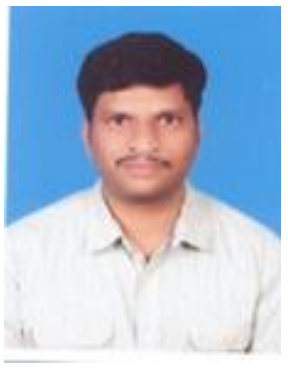

Dr. Srinivasa Reddy K received the B.Tech. (CSE) degree from JNTU, Hyderabad in 2003 , Masters Degree in M.Tech. from SRM University, Chennai, T.N., India in 2005. He received Ph.D. in Faculty of Computer Science \& Engineering from JNTUA, Anantapuram, A.P., India, in 2016. At present, he is working as Professor in Institute of Aeronautical Engineering, Dundigal, Hyderabad, T.S, India. His research interests include Image Processing, computer vision, and machine learning. He has published research papers in various National, International conferences and Journals. He is a life member of ISTE, CSI. 


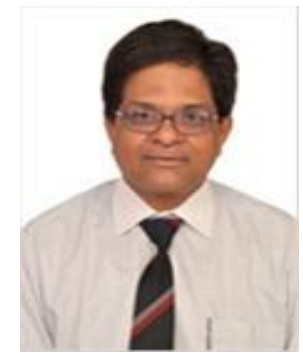

Dr. Vijaya Kumar $\mathbf{V}$ is working as Dean in Dept. of CSE \& IT and Director Centre for Advanced Computational Research (CACR) at Anurag Group of Institutions, (AGOI) (Autonomous), Hyderabad. He received integrated M.S.Engg, in CSE from USSR in 1989. He received his Ph.D. degree in Computer Science from Jawaharlal Nehru Technological University (JNTU), Hyderabad, India in 1998 and guided 24 research scholars for Ph.D. He has served JNT University for 13 years as Assistant Professor and Associate Professor. He has received best researcher and best teacher award from JNT University, Kakinada, India. His research interests include Image Processing, Pattern Recognition, Digital Water Marking, Cloud Computing, Image Retrieval Systems and image analytics in Big Data. He is the life member of CSI, ISCA, ISTE, IE (I), IETE, ACCS, CRSI, IRS, and REDCROSS. He published more than 125 research publications till now in various National, International journals and conferences. He has also established and also acted as a Head, Srinivasa Ramanujan Research Forum (SRRF) at GIET, Rajahmundry, India for promoting research and social activities.

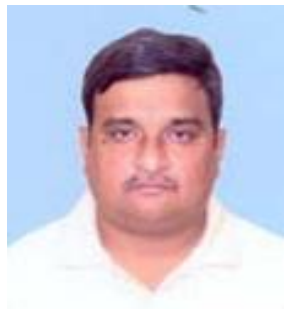

Dr. Venkata Krishna V received the B.Tech. (ECE) degree from Sri Venkateswara University. He completed his M. Tech. (Computer Science) from JNT University. He received his Ph.D. degree in Computer Science from Jawaharlal Nehru Technological University (JNTU), Hyderabad, India in 2004. He worked as Professor and Head of the Department for 10 years in Mahatma Gandhi Institute of Technology, Hyderabad. He worked as Principal for Vidya Vikas College of Engineering, JNTU, Hyderabad, Chaitanya Institute of Science \& Technology, JNTU, Kakinada. Godavari Institute of Engineering and Technology, Rajahmundry, JNTU, Kakinada. At present He is working as Professor, CSE Department in Vidya Jyothi Institute of Technology, Hyderabad, India. He is an advisory member for many Engineering colleges. He Published more than 40 research publications in various National, International journals and conferences. Guided 4 research scholars for Ph.D. He is a life member of ISTE and CSI. 